\title{
Au-delà des « Natacha » : les migrations féminines postsoviétiques à Istanbul
}

Beyond "Natasha": Women Migrations from Post-Soviet Countries in Istanbul Más allá de las «Natacha»: las migraciones femeninas postsoviéticas en

Estambul

\section{Adeline Braux}

\section{OpenEdition}

Journals

\section{Édition électronique}

URL : https://journals.openedition.org/remi/7195

DOI : $10.4000 /$ remi.7195

ISSN : $1777-5418$

Éditeur

Université de Poitiers

Édition imprimée

Date de publication : 1 janvier 2015

Pagination : 81-102

ISBN : 979-10-90426-24-5

ISSN : 0765-0752

Référence électronique

Adeline Braux, «Au-delà des « Natacha » : les migrations féminines postsoviétiques à Istanbul », Revue européenne des migrations internationales [En ligne], vol. 31 - $n^{\circ} 1$ | 2015, mis en ligne le 01 janvier 2018, consulté le 14 avril 2022. URL : http://journals.openedition.org/remi/7195; DOI : https://doi.org/ 10.4000/remi.7195 


\section{Au-delà des " Natacha ": les migrations féminines postsoviétiques à Istanbul}

\section{Adeline Braux ${ }^{1}$}

Lorsque les frontières anciennement turco-soviétiques se sont ouvertes à la faveur de la disparition de I'URSS en 1991, plusieurs figures ont émergé symbolisant jusqu'à présent les mobilités régionales. Les tchelnoki originaires de I'ancien bloc socialiste, appelés également "commerçants à la valise $"^{2}$ ou " navetteurs ", ont ainsi longtemps fait partie du paysage sur les marchés stambouliotes. La situation socio-économique s'améliorant dans leur pays d'origine, ils ont peu à peu cédé la place aux commerçants et commerçantes du Maghreb ou d'Asie centrale. Une autre figure familière de cette époque riche en mobilités tous azimuts est la "Natacha ", comme on appelle en Turquie les prostituées originaires de I'ex-URSS. Leur présence a défrayé la chronique, surtout à Istanbul et dans les villes situées sur la mer Noire (Bilici, 1994 : 167-171), et a donné naissance à des stéréotypes tenaces sur les femmes originaires d'Europe de l'Est. La notion d' "Europe de l'Est " doit en l'occurrence être appréhendée au sens large puisque les femmes concernées sont originaires tant de Russie, d'Ukraine ou de Moldavie que du Caucase du Sud, voire d'Asie centrale depuis quelques années. La prostitution est toutefois loin d'être la seule activité économique de ces migrantes, qui ont également investi le secteur du commerce et, plus récemment, celui des services à la personne. Les migrations des femmes ex-soviétiques vers la Turquie, que se propose d'étudier cet article, représentent un défi au présupposé selon lequel les "pays du Nord " seraient caractérisés par la crise du care et le vieillissement de la population et attireraient les femmes des "pays du Sud ${ }^{3}$. Les pays dont sont originaires les migrantes se situent, d'un point de vue géographique, au nord de la Turquie mais, d'un point de vue purement économique, la Turquie fait figure de Nord pour les ressortissantes

1 Responsable de l'Observatoire du Caucase (Bakou), Institut français d'études anatoliennes (IFEA-Istanbul), ambassade de France en Azerbaïdjan, 13 rue Louveau, 92438 Chatillon cedex; adelinebraux@hotmail.com

2 Bavul ticareti en turc, littéralement " commerce à la valise ".

3 Le présent article s'appuie sur un travail de terrain engagé, à Istanbul, en 2012, auprès de femmes originaires des pays du Caucase du Sud (Arménie, Azerbaïdjan et Géorgie), ainsi que de Russie et d'Ukraine dans le cadre de deux projets de recherche intitulés "Les migrations ex-soviétiques en Turquie : des nouvelles formes de circulation migratoire dans l'espace eurasiatique " et "Caucase du Sud : recompositions plurielles d'un champ migratoire ". L'enquête a également concerné, de façon marginale, plusieurs femmes originaires d'Ouzbékistan et du Turkménistan. 
des pays de départ concernés, en raison du boom économique que connaît le pays depuis le début des années 2000. En ce sens, ces formes de migrations donnent lieu à des connexions inédites et permettent de déconstruire une vision eurocentrique des migrations au sein de laquelle la dichotomie Nord/Sud paraît de moins en moins pertinente.

II nous faut aussi constater que s'intéresser aux migrations féminines ex-soviétiques en Turquie revient, in fine, à reconnaître la possibilité d'une spécificité des acteurs de sexe féminin en migration. Émerge dans ce cas, un paradoxe immuable à la qualité de femme-migrante : comment concilier la décision symboliquement et socialement très forte d'émigrer avec les charges de famille (maternelles ou autres) qui pèsent sur leurs seules épaules ? Peut-on parler d' "empowerment " et de processus d'autonomisation dès lors que la migration est inextricablement liée à des contraintes familiales prégnantes, quand celles-ci ne sont pas à l'origine de la première ? L'hétérogénéité des situations migratoires et la diversité des parcours rendent néanmoins une analyse purement binaire sur le mode autonomisation/dépendance peu opérante pour tenir compte de la complexité de la situation personnelle et migratoire de chacune. Nous verrons que les mobilités féminines postsoviétiques en Turquie prennent des formes variées, qui se recoupent parfois, et racontent toutes un rapport particulier à la migration.

\section{La Turquie au cœur des migrations régionales}

Avec la disparition de I'URSS sont apparues des frontières, qui n'existaient pas auparavant, entre les anciennes républiques soviétiques. Parallèlement, d'autres frontières sont devenues beaucoup plus faciles à franchir, spécialement entre l'ex-URSS et la Turquie qui renouaient ainsi des liens tissés de longue date. En cherchant à rallier les mers chaudes, dont l'Empire russe était privé à cause de sa situation géographique, les tsars ont, de fait, été confrontés aux Empires ottoman et perse. Malgré les guerres, les destructions, les pillages et les déplacements de population, l'Eurasie n'a jamais cessé de communiquer et d'échanger des biens, des personnes et des savoirs. L'existence de mouvements de population protéiformes et anciens explique la présence actuelle, en Russie, de populations d'origine turcique et, en Turquie, de diasporas caucasiennes, mais également en Géorgie celle de populations islamisées et sur les côtes pontiques du nord de la Turquie de minorités de langue géorgienne. La création de l'Union soviétique a subitement mis un terme à ces contacts pluriséculaires en entérinant l'arrimage de laTurquie, majoritairement asiatique d'un strict point de vue géographique, au camp occidental.

La Turquie a longtemps été un pays d'émigration, en raison, notamment, des accords bilatéraux passés avec l'Allemagne qui ont été à l'origine de I'installation de nombreux gastarbeiter dans ce pays, rejoints par la suite par leur famille ${ }^{4}$. À cette émigration de travail s'ajoutent les milliers de personnes réfugiées pour des motifs politiques suite au coup d'État de 1980 et au conflit

4 Depuis 2006, le mouvement s'est inversé puisque la troisième génération issue de l'immigration turque a fait le chemin inverse, à tel point qu'en 2009 le solde migratoire entre la Turquie et l'Allemagne a été favorable à la première (Basak Serap Uzun, 2011). 
entre l'armée turque et le Parti des travailleurs du Kurdistan (PKK). Depuis le début des années 1990 toutefois, la situation géographique et géopolitique du pays, carrefour géopolitique du grand Moyen-Orient, a transformé la Turquie, et spécialement Istanbul, en une sorte de hub pour les migrants en route vers les pays européens, originaires de pays ou de régions lointains comme le Bangladesh ou la Corne de l'Afrique. Pour ces migrants, la Turquie est un pays de transit qui devient parfois un pays d'installation, comme c'est le cas pour les personnes dont la demande d'asile a été rejetée par les autorités turques (OIM, 2008 : 31). Pour les ressortissants des pays d'Europe de l'Est et de l'ancien bloc soviétique en revanche, la Turquie est apparue dès la chute du Rideau de fer, voire un peu avant ${ }^{5}$, comme une destination possible à défaut de représenter un pays d'immigration durable. Ces migrations ont d'emblée pris un caractère circulaire en raison de la facilité avec laquelle les uns et les autres peuvent se rendre de part et d'autre de la frontière, à l'opposé de I' " obsession des frontières " (Foucher, 2012) et à son corollaire sécuritaire qui caractérisent nombre de frontières dans cette zone. Si les frontières occidentale (fleuve Evros/Grèce) et méridionale (" questions " kurde et syrienne) de la Turquie constituent des - ou bien ont été constituées en - problèmes sécuritaires, la situation semble moins ardue aux frontières avec l'ex-URSS. La frontière terrestre avec l'Arménie représente certes une exception notable mais il s'agit davantage d'une question $d^{\prime}$ 'ordre politique que d'ordre sécuritaire, même s'il est vrai que le politique et le sécuritaire sont corrélés. Nombreux d'ailleurs, sont ceux au niveau local dans la province de Kars - frontalière de l'Arménie - qui réclament l'ouverture pure et simple de la frontière avec l'Arménie.

La politique turque en matière de gestion des flux migratoires est très influencée par la volonté des autorités de se conformer à l'acquis communautaire en vue d'une adhésion, fût-elle de plus en plus hypothétique, à I'Union européenne. Le pays s'est donc doté progressivement d'un grand nombre d'instruments juridiques et institutionnels afin de gérer les flux migratoires. La première loi nationale relative au séjour des étrangers (Ikamet ve seyahatleri hakkında kanun) date de juillet 1950. Le pays a par ailleurs ratifié la Convention de Genève de 1951 sur les réfugiés, ainsi que son protocole additionnel de 1967, et a signé un certain nombre de textes bilatéraux. La Direction générale des migrations (Göç Idaresi Genel Müdürlügü) est compétente. Elle est placée sous la tutelle du ministère de l'Intérieur, pour les questions liées aux étrangers et s'est dotée de moyens modernes pour faire face à l'afflux de demandes de documents administratifs. Les rendez-vous pour l'obtention des documents de séjour se prennent dès lors par internet et les bureaux des étrangers de la police de chaque district sont sollicités pour les extensions de permis de séjour (oturma izni). La dernière "Loi sur les étrangers et la protection internationale " (Yabancılar ve uluslararası koruma kanunu, loi N6458) a été adoptée le 4 avril 2013 par l'Assemblée nationale de Turquie. L'adoption de cette loi parachève à bien des égards une forme de transition pour la Turquie dans le domaine migratoire. La question du séjour est du reste au cœur de cette nouvelle loi. Celle-ci dispose que les étrangers entrés sur le territoire turc (soumis ou non à l'obligation d'obtenir un visa au préalable) ne peuvent plus séjourner dans le pays plus de 90 jours d'affilée sur une période de 180 jours (article 11). Il s'agit

5 La présence de Polonais notamment a été rapportée dès le milieu des années 1980. 
là d'une modification substantielle puisqu'auparavant la durée limite de séjour était également limitée à quatre-vingt-dix jours mais sans condition de durée à long terme. Dans la même veine, I'article 13 dispose que la délivrance des visas aux frontières devient exceptionnelle alors qu'il s'agissait jusqu'à présent d'une pratique assez courante qui assurait souplesse et fluidité. En outre, la loi énumère les six types de titres de séjour existant (article 30) : permis de séjour de courte durée ; permis de séjour à titre familial (semblable à la carte de séjour "vie privée et familiale " en France) ; permis de séjour pour les étudiants ; permis long séjour ; permis de séjour à titre humanitaire ; permis de séjour pour les victimes de trafic d'êtres humains. L'harmonisation de la législation turque avec les standards européens apparaît ainsi de façon paradoxale comme étant moins favorable aux étrangers dans certains domaines, même si le volet " protection internationale " a le mérite de fixer un certain nombre d'obligations pour la Turquie en matière d'asile.

Nombre de pays issus de l'ex-URSS, dont les pays du Caucase du Sud (Arménie, Azerbaïdjan et Géorgie) et d'Asie centrale (Kirghizstan, Ouzbékistan et Tadjikistan en premier lieu), sont caractérisés par une forte émigration, la Russie demeurant leur premier choix de destination. Ces mobilités sont d'autant plus facilitées que la majorité des ressortissants des anciennes républiques soviétiques peuvent se rendre en Russie sans visa. Elles ont, en outre, pour point commun d'être des migrations essentiellement masculines. Or, force est de constater qu'en Turquie, la situation s'inverse puisque les migrants originaires d'ex-URSS, y compris des pays d'Asie centrale et du Caucase, sont pour la plupart des migrantes. II en va de même pour l'émigration en provenance de Russie, d'Ukraine et de Moldavie. D'après I'Institut des statistiques de Turquie (Türkiye statistikler Kurumu), l'immigration légale de ressortissants originaires de l'ex-URSS s'est élevée à un peu plus de 30000 personnes en 2012. À l'exception des Arméniens et des Moldaves, dont l'entrée sur le territoire turc est soumise à l'obtention d'un visa qu'ils peuvent se procurer sans difficultés majeures, les ressortissants azerbaïdjanais, géorgiens, russes, ukrainiens, ainsi que ceux de I'ensemble des pays d'Asie centrale peuvent se rendre en Turquie sans visa, voire munis de leur seule carte d'identité dans le cas des Géorgiens ${ }^{6}$. Les statistiques concernant les entrées de ressortissants de l'espace post-soviétique sur le territoire turc donnent une idée des flux : en 2006, il y aurait eu pas moins de 1790041 entrées de citoyens russes en Turquie et 451256 entrées de citoyens ukrainiens. Pour les ressortissants arméniens, azerbaïdjanais et géorgiens, ce chiffre était respectivement de $38173 ; 347906$ et $498163^{7}$. En 2011, la Fédération de Russie (près de 3500 000, 11 \% du total) et la Géorgie (1 $000000,3 \%)$ figuraient respectivement à la deuxième et à la huitième place du " top 10 " du nombre d'entrées en Turquie par nationalité ${ }^{8}$. Une fois entrés sur le territoire turc, ils peuvent y séjourner de 30 à 90 jours. Par conséquent,

6 La Géorgie est le seul pays extra-communautaire, à l'exception de la Suisse, dont les ressortissants bénéficient de cette facilité.

7 Statistiques compilées obtenues après du service des étrangers du ministère de I'Intérieur turc et de I'Institut national des statistiques, citées par Kemal Kirisci, Informal 'Circular migration' Into Turkey: The Bureaucratic and Political Context, CARIM 21, 2008 : 13. II va sans dire que le même individu peut franchir à plusieurs reprises la même frontière.

8 Source ministère turc du Tourisme : http://www.kultur.gov.tr/EN,36568/number-of-arriving-departing-foreigners-and-citizens.html, page consultée le 3 janvier 2013. 
nombre de migrantes qui se rendent en Turquie avec un simple statut de touriste n'entament pas nécessairement des démarches de droit au séjour et travaillent de façon illégale. Pour celles qui s'y soumettent, elles obtiennent dans la plupart des cas une autorisation de travail (çalısma izni) d'un mois. La législation a toutefois été modifiée en février 2012 : une fois le visa de 90 jours écoulé, les étrangers sont tenus de quitter le pays et ne peuvent pas revenir en Turquie avant trois mois. Ce changement législatif est cependant générateur de difficultés pour les Géorgiens, qui sont très mobiles entre les deux pays.

Les circulations migratoires dont il est question ont de surcroît pour particularité d'avoir été, dès l'origine, conjuguées au féminin et de ne pas être la conséquence d'une immigration qui aurait été en premier lieu masculine, puis familiale. Cette immigration féminine donne, de plus, rarement lieu à un processus qu'on pourrait qualifier de "regroupement familial inversé ", qui verrait les migrantes faire venir leur conjoint et leurs enfants une fois installées ${ }^{9}$. Tout porte donc à croire que "le genre compte " (" gender matters ") (Mahler et Pessar, 2006 : 27-63). Si le processus migratoire est générateur d'autonomisation et de responsabilisation pour ces femmes au sein de la cellule familiale, voire au-delà dans le cercle communautaire local, l'autonomisation qui en découle reste néanmoins limité dans la mesure où ces migrantes demeurent dépendantes de leurs obligations familiales, à l'exception notable de celles qui se rendent en Turquie car elles ont contracté une union avec un ressortissant turc. Ces femmes quittent leur pays par nécessité, et non pas pour " partir à l'aventure ", et ce tout en sollicitant l'accord de leur famille. Une fois en migration, le soupçon de prostitution à l'encontre de celles qui sont pourtant le seul soutien de famille de leur famille n'est, comme nous le verrons, jamais loin.

\section{Du commerce à la valise aux services à la personne et à I'entertainement business}

Les recherches consacrées à ces circulations migratoires féminines en Turquie ont majoritairement une finalité opérationnelle et/ou ne s'intéressent pas spécifiquement à leur caractère féminin. L'“ occultation des femmes migrantes " (Leandro, $2006: 18$ ) n'est néanmoins pas une spécificité de l'étude des migrations en Turquie. Bien que les femmes aient toujours constitué une part non négligeable des populations en migration, elles n'ont été que peu étudiées jusqu'à une période récente, car longtemps associées au processus de regroupement familial ${ }^{10}$. Elles représentent pourtant bien plus qu'une simple affaire de franchissement de frontières étatiques par un individu isolé (Benhabib et Resnik, 2009 : 13). Un rapide tour d'horizon sur les études réalisées sur ce sujet s'attachent à des secteurs d'activité présentés comme étant traditionnellement féminins : entretien, services à la personne, etc. (Catarino et Morokvasic, 2012 : 7-27). L'un des écueils des études sur les migrations dites "féminines" a été, à force de se concentrer sur l'acteur "femme ", d'oublier les hommes et, par conséquent, d'occulter l'ensemble du maillage social au sein duquel les

9 Ce qui a pu être le cas des migrantes philippines aux États-Unis, par exemple (Le Espiritiu, 2005 : 9).

10 La production en sciences sociales consacrée aux thèmes du genre et de la migration est abondante depuis une quinzaine d'années (Cossée et al., 2012: 14). 
migrantes évoluent. Dans le même temps, la tendance à envisager les migrations féminines par le prisme des rapports de domination entre les sexes a débouché sur ce que d'aucuns qualifient de "victimisation " des femmes migrantes. Nous posons toutefois que l'une et l'autre approches se justifient et ne sont du reste pas incompatibles, même si certains ont souligné que la recherche sur les migrations féminines tend à être supplantée par une analyse en termes de genre (Donato, Gabaccia et al., $2006: 39)^{11}$.

L'un des secteurs d'activités les plus prisés par les migrantes ex-soviétiques en Turquie a été pendant longtemps celui du commerce à la valise. Ces activités s'inscrivent dans un contexte qui a vu émerger la figure de "la commerçante ", en particulier, en Europe du Sud (Schmoll, 2006 : 3). À I'instar de leurs homologues masculins, les navetteuses ne font pas partie des individus les plus pauvres et défavorisés de la société d'où ils viennent : elles appartiennent la plupart du temps à la classe moyenne et sont originaires des pays engagés dans une période de transition engendrant une grande instabilité socioéconomique. Comme les hommes, les commerçantes à la valise d'hier sont, pour quelques unes d'entre elles, devenues des petits entrepreneurs relativement prospères voire des businesswomen reconnues (Kirisçi, 2008 : 4). Certains parcours migratoires débutés au plus bas de l'échelle sociale se sont achevés en success stories: telle femme qui " navettait " comme commerçante à la valise entre la Russie et la Turquie sera devenue une femme d'affaires aguerrie, telle Azerbaïdjanaise embauchée comme simple guide touristique se trouve à la tête d'une agence de voyages florissante. Ces succès ont été en partie facilités par I'acquisition de la citoyenneté turque grâce à un mariage " blanc ", d'autant que jusqu'au début des années 2000 les étrangers qui contractaient une union avec un(e) ressortissant(e) turc (turque) se voyaient accorder très facilement la nationalité turque. Par la suite, les conditions se sont durcies ; les contrôles demeurent cependant moins stricts que dans les pays de I'Union européenne ou aux États-Unis. Ceci pose naturellement la question du devenir du projet migratoire et de la promotion sociale qui lui est associée. Il s'agit là d'une interrogation qui concerne il est vrai tant les hommes que les femmes mais, lorsqu'il est question de ces dernières, elle permet de sortir de l'image de la femme employée à des tâches dites de reproduction sociale et d'esquisser les contours des initiatives entrepreneuriales des migrantes. Traditionnellement, le rôle des femmes dans le secteur des affaires est minoré, les valeurs entrepreneuriales de courage et de risque étant bien plus volontiers associées aux hommes (Basu et Werbner, $2009: 53$ ).

Longtemps lieu d'approvisionnement exclusif des commerçantes à la valise russes, ukrainiennes et polonaises, dans les années 1990/2000, le quartier de Laleli - situé entre Aksaray, interface de transport centrale à Istanbul, et le quartier touristique de Sultanahmet - a connu des fortunes diverses au gré des crises économiques et financières en Turquie et dans les pays de I'ancien bloc soviétique. Cette zone d'achalandage demeure un lieu quasiment incontournable pour les commerçantes à la valise, même si d'autres zones de vente ont émergé.

11 Sarah J. Mahler et Patricia R. Pessar (2006 : 31) expliquent même ce qu'elles qualifient de " marginalisation du genre " dans les études migratoires par le caractère foncièrement qualitatif des recherches conduites dans ce domaine. 
Par exemple à Osmanbey ${ }^{12}$, où on trouve essentiellement du prêt-à-porter de qualité supérieure à celui de Laleli, ou bien dans des quartiers plus éloignés du centre mais situés à proximité des ateliers de confection, comme à Yenibosna. Jusqu'à présent, le succès des produits textiles turcs, il est vrai fortement concurrencés par les produits chinois, ne se dément pas. Il est ainsi très fréquent de voir proposés dans des marchés situés aux quatre coins de l'ex-URSS les mêmes blouses pour femmes, ou les mêmes vêtements pour enfants que ceux présentés à Laleli. À Aksaray, par exemple, l'offre de services aux russophones, et de plus en plus aux arabophones, est variée (des téléphones portables aux produits d'hygiène) et la langue russe très usitée. Des observations de terrain, en novembre 2013, ont permis de constater la présence de plus en plus notable de femmes originaires d'Asie centrale. Mères voire grands-mères pour la plupart, elles sont accompagnées de jeunes filles. Comme les commerçantes tunisiennes (Schmoll, 2005), elles se déplacent en " grappes " et sont aisément reconnaissables à leurs vêtements et à leur foulard noué sur le haut du crâne laissant la nuque et le visage dégagés.

Parmi les secteurs économiques traditionnels investis par les migrantes ex-soviétiques, on trouve, dans le tertiaire, celui des services à la personne (auxiliaires de vie, gardes d'enfants et femmes de ménage surtout). De ce point de vue, ces migrations ne se distinguent guère de celle des femmes d'Europe de I'Est en Italie (D’Ottavio, 2005 : 89-103; Ambrosini, 2005 : 71-88). Le secteur des services, et plus précisément celui des services à la personne, a connu une croissance soutenue dans les grandes villes turques depuis la libéralisation de l'économie dans les années 1980. De surcroît, avec la croissance économique des années 2000, de plus en plus de familles turques ont des revenus qui leur permettent d'avoir des employés de maison. Compte tenu des évolutions démographiques que connaît le pays, la demande dans ce secteur devrait aller croissant. Le taux de natalité tend à se stabiliser à 2,08 enfants par femme, même si de fortes disparités persistent dans le pays ${ }^{13}$. $\mathrm{D}^{\prime}$ après les projections de I'Institut des statistiques de Turquie, à I'horizon 2023 le pays compterait 2,4 millions de personnes de plus de 85 ans (pour une population de 84 millions d'habitants), près de 3 millions (sur 94 millions) en 2050. En somme, les conditions structurelles dans le pays d'installation semblent réunies pour rencontrer les vocations migratoires émanant de par-delà les frontières nationales. En Turquie subsiste une forte "culture du maintien à domicile " (Ambrosini, 2005 : 83), par conséquent l'embauche d'une femme qui demeurera en permanence au domicile de la famille apparaît comme une solution préférable à un placement dans une institution spécialisée, structure encore peu répandue, l'usage voulant que les parents soient pris en charge par les enfants (en général chez un des fils). Autrefois philippines, les garde-malades sont actuellement originaires d'ex-URSS (du Turkménistan et de Géorgie). Les premières sont appréciées car elles maîtrisent une langue proche du turc de Turquie ${ }^{14}$. Force est néanmoins de constater que les Géorgiennes sont très représentées dans ce secteur. De ce

\footnotetext{
12 Quartier aisé et relativement central situé dans le district de Sisli.

13 Dans la région "Marmara ouest ", le taux de natalité s'établit à 1,61, tandis que dans la région " sud-est ", il s'établit à 3,47.

14 Les linguistes distinguent quatre sous-groupes parmi les langues turciques. Le turc de Turquie, le turkmène et l'azéri se trouvant dans le même sous-groupe, il y a en partie intercompréhension entre les locuteurs de ces langues.
} 
point de vue d'ailleurs, la situation paraît également quelque peu inhabituelle puisque la Turquie est encore relativement dynamique d'un point de vue démographique au regard des standards européens, alors que la Géorgie connaît une crise démographique profonde depuis une vingtaine d'années.

Le processus migratoire est le même que celui que l'on observe sous d'autres latitudes : une connaissance, une amie ou une parente plus ou moins proche fait part de l'existence de places vacantes aux candidates potentielles. Les premières femmes arrivées ont servi d'éclaireurs aux suivantes, à la différence de ce qui a pu être observé dans d'autres situations où les migrantes qui se rendaient à l'étranger pour travailler rejoignaient un époux, un père, ou un frère ${ }^{15}$. Des agences de recrutement et de placement ont vu le jour (Kirisçi, 2008 : 5), auxquelles s'adressent les familles en quête d'une nounou ou d'une auxiliaire de vie. Pour les femmes salariées dans le secteur des services à la personne, il s'agit, dans la plupart des cas, d'une reconversion complète puisqu'elles exerçaient auparavant des métiers aussi divers que comptable ou ingénieur dans le bâtiment. De ce point de vue l'activité professionnelle et le salariat ne sont pas une nouveauté pour celles qui exerçaient un métier dans leur pays d'origine. Le salaire est d'environ 600 dollars par mois pour un travail à plein temps, certaines migrantes percevant en outre une somme de 30 lires turques environ (soit environ 8 euros) pour financer, le cas échéant, les trajets en transports en commun (yol parası). Leurs activités dépassent largement le cadre de la seule compagnie à des personnes âgées et exigent une certaine polyvalence. Outre les conversations, les promenades et les travaux ménagers, les migrantes sont fréquemment appelées à remplir des fonctions quasiment paramédicales puisqu'elles sont amenées à administrer des médicaments et à veiller sur des personnes atteintes de pathologies pas toujours liées à la vieillesse. Les liens tissés sont d'autant plus forts que l'auxiliaire de vie est logée au sein même de la demeure familiale, fréquemment assez spacieuse pour accueillir une chambre pour elle, voire une studette équipée d'une salle de bain et d'une kitchenette avec tout le confort. La plupart des femmes rencontrées sur le terrain sont employées dans les quartiers cossus de la rive asiatique (Kadiköy, Moda, Yeditepe), plus calmes que la rive européenne, et où il fait bon flâner le samedi, unique jour de repos. Les relations deviennent vite familières (Ambrosini, 2005 : 85) et vont bien au-delà de la simple prestation de service attachée au contrat conclu. Ceci n'est d'ailleurs pas sans provoquer des jalousies au sein des familles : telle fille reprochera par exemple à sa mère d'être plus attentive à l'auxiliaire de vie qu'à elle-même. Installée à demeure chez la personne âgée dont elle a la responsabilité, elle est à la fois garde-malade, confidente, infirmière, cuisinière, dame de compagnie ${ }^{16}$. Conviée aux fêtes familiales, sollicitée pour rendre visite à la personne âgée lorsque celle-ci est hospitalisée, elle acquiert à certains égards une autorité, qui s'apparente à une forme de soft power. Les liens presque familiaux entretenus avec les employeurs se prolongent au-delà de la disparition

15 Processus typique dans le cas des migrations de femmes portugaises (Leandro, $2006: 22)$.

16 La communication est d'autant plus aisée que les migrantes acquièrent au fil du temps les compétences linguistiques nécessaires. La maîtrise, même basique, du turc revêt en l'occurrence un caractère indispensable. Les migrantes qui maîtrisent le russe et dont la langue maternelle est éloignée du turc (les Arméniennes et les Géorgiennes) utilisent des méthodes d'apprentissage du turc rédigées en russe. Les rayons "langues " des librairies d'Istanbul sont du reste bien achalandés en la matière. 
de la personne âgée car certaines migrantes maintiennent des relations avec les enfants de celle-ci.

Si cet article se propose d'aller au-delà des clichés véhiculés habituellement sur les migrantes originaires de l'ex-URSS, il invite également à considérer, sinon sous un angle nouveau, du moins sous un angle plus large, l'épineuse question de la prostitution et de la " traite des femmes ${ }^{17}$. Ce domaine de recherche a été envisagé essentiellement par le prisme de la violence exercée à l'encontre des femmes, ce qui a pu conduire à négliger d'autres aspects. Dans une étude consacrée à la traite des femmes originaires d'Europe de l'Est en Italie, Rutvica Andrijasevic (2005 : 155-175) déplace le curseur de son analyse sur les liens entre prostitution d'une part et migrations et travail d'autre part dans un contexte d'évolution de la législation européenne sur l'immigration. La prise en compte du contexte dans lequel s'inscrivent ces activités semble aussi indispensable qu'une mise en perspective du projet migratoire et de la recherche de mobilité sociale qui lui est inhérente. Associées aux activités de commerce (Guillemaut, 2008 : 95), la prostitution peut représenter, tout au plus, un revenu d'appoint sans être systématiquement liée à des formes d'exploitation. Un rapport de I'Organisation internationale pour les migrations datant de 2003 (Erder et Kaska) soulignait ainsi que, si la Turquie est le principal pays de destination des femmes victimes de la traite originaires d'Azerbaïdjan, de Géorgie, de Russie et d'Ukraine ${ }^{18}$, toutes ne sont pas venues à la prostitution par le biais de réseaux. De même, si nombre de jeunes femmes ont été trompées par des intermédiaires sur la nature des activités professionnelles promises à Istanbul, certaines s'y sont rendues en toute connaissance de cause ${ }^{19}$. Mais, de fait, la tendance à maintenir la focale exclusivement sur le statut de victime des femmes concernées dans le processus de traite tend à rendre inopérante toute tentative de décryptage des motivations individuelles à l'œuvre dans le cadre plus global de la traite (Andrijasevic, $2005: 3$ ).

Nous évoquions dans l'introduction les conséquences de l'arrivée dans les régions turques de la mer noire de prostituées originaires de l'ex-URSS, notamment dans les foyers turcs, au sein desquels les cas de divorces se sont multipliés dans les années 1990 (Bilici, 1992). Dans une veine tantôt comique, tantôt dramatique, miroir des problématiques sociétales, le cinéma s'est en partie emparé du sujet des " Natacha ». En 2008, le réalisateur Özcan Alper, dans son film Sonbahar ${ }^{20}$ (" Automne "), relatait une histoire d'amour entre un ancien détenu politique et une prostituée géorgienne. Dans une scène, les deux

17 Pour I'ONU, ce terme désigne le fait de « recruter, transporter, transférer, héberger ou accueillir une personne en ayant recours à la force, à la contrainte, à la tromperie ou à $d^{\prime}$ 'autres moyens, en vue de l'exploiter ". Lorsque les activités de traite d'êtres humains concernent les femmes, la corrélation avec le travail sexuel est très forte. La prostitution n'étant pas la seule forme d'exploitation à laquelle les femmes sont exposées, certaines voix s'élèvent en faveur d'un élargissement de la problématique et préconisent d'établir une distinction entre traite et travail forcé (Erder et Kaska, $2003: 9-10$ ).

18 La Moldavie et la Roumanie étaient également concernées.

19 Dans ce cas toutefois, elles sont régulièrement confrontées à leur arrivée à des conditions de travail et d’hébergement caractérisées par une grande précarité.

20 Le film se déroule dans la région d'Artvin, dans un village hamchène, du nom d'une minorité arménienne islamisée du nord-est de la Turquie. Youssouf, condamné à une peine de prison en 1997 suite à sa participation à des manifestations estudiantines, retourne dans son village d'origine après dix ans de détention. 
principaux protagonistes se rencontrent dans une librairie où la jeune femme achète un roman de littérature russe classique. Elle paie puis quitte le magasin. Le jeune homme passe à son tour à la caisse et le vendeur lui lance alors tout de go : "Les Russes, même leurs putes sont cultivées ! ". Et il est vrai que cette réplique pour le moins abrupte reflète une certaine réalité puisque les migrantes ex-soviétiques qui travaillent en Turquie sont majoritairement éduquées. Ce sont aussi bien souvent des mères de famille, voire de jeunes mères de famille, qui élèvent leurs enfants, même à distance, conformément aux normes sociales. La mise en scène de leur corps liée à leur activité a pour conséquence d'occulter les autres traits de leur personnalité, de leur identité et de leurs aspirations (Gil, 2008 : 24), comme si lire de la littérature en russe était incompatible avec la prostitution.

Les histoires de jeunes femmes à qui on avait promis un emploi de serveuse circulent parmi les migrantes ; "ça se dit ", mais, personne ne connaît personnellement les personnes concernées. Lorsqu'il s'agit de prostitution volontaire, les faits sont évoqués sans que le mot " prostituée " ne soit jamais prononcé. Dans le pays d'origine et dans les familles surtout, le soupçon n'est jamais loin :

"Quand j'ai dit à mon frère que j'allais partir en Turquie, il m'a répondu : "Quand les femmes partent, c'est qu'il y a des hommes". II avait peur que je fasse ça, comme les Turkmènes et les Ouzbèkes ${ }^{21}$. "

(Nino, Istanbul, janvier 2013)

Le secteur de l'industrie du loisir est toujours évoqué lui aussi à mots couverts tant les frontières avec le milieu de la prostitution apparaissent poreuses. Par exemple Nelly, originaire de Tachkent, expliquait, dans un russe qui rendait ses origines indécelables, qu'elle avait été employée comme femme de chambre dans un hôtel à son arrivée à Istanbul en 2009. Elle jugeait que le salaire était très insuffisant compte tenu des contraintes horaires et de la fatigue physique engendrée par des tâches qui étaient ingrates ; et, ajoutait-elle pudiquement, elle avait décidé de travailler par la suite " dans une autre sphère " ("v drugoj sfere ", en russe), à savoir comme barmaid dans un établissement du quartier de Maltepe, sur la rive asiatique. La nature exacte de ses activités est certes demeurée incertaine à l'issue de l'entretien, néanmoins les éléments non verbaux et contextuels, dont la présence lors de l'entretien d'un homme qu'elle présentait comme son patron, laissait penser que son rôle ne se limitait pas à celui de serveuse ${ }^{22}$. Cet exemple illustre que la duperie quant aux conditions d'exercice professionnel n'est pas nécessairement à l'origine de la prostitution,

Il est accueilli par sa mère, âgée et malade, demeurée seule au village après le décès de son mari. La sœur de Youssouf a déménagé dans la ville voisine. Ne reste que Mikail, un ami de Youssouf. En ville, Youssouf fait la connaissance d'une jeune femme géorgienne, Eka, qui travaille comme prostituée et a laissé, en Géorgie, sa fille à la garde de sa mère. Isolés chacun dans leur monde, Youssouf et Eka feront un bout de chemin ensemble.

21 Le préjugé rejoint en partie la réalité puisque, parmi les personnes contraintes à se prostituer, les jeunes femmes ouzbèkes et turkmènes issues du milieu rural sont au cœur des préoccupations des organisations turques et internationales.

22 La rencontre avait pu être organisée par l'intermédiaire d'un entrepreneur du bâtiment retiré de ce qu'il appelait ce " milieu " et de ce " mode de vie ". L'entretien avait eu lieu à deux pas de l'établissement où travaillait Nelly, situé lui-même à proximité de son logement, dans un quartier résidentiel qu'elle connaissait bien. II s'était déroulé en russe et la " patron " en question n'avait pas pu le comprendre. 
ou des activités qui flirtent avec la prostitution. En l'occurrence, Nelly a choisi de changer de profession de son plein gré parce qu'elle y voyait un moyen de gagner autant d'argent, voire plus, dans des conditions qui la satisfaisaient, tout en s'offrant de nouvelles opportunités et en ayant plus d'autonomie. Son cas n'est à cet égard guère différent de celui de nombre de migrantes rencontrées, qui ont pour point commun d'être des femmes seules, libérées de ce qu'elles présentent comme le carcan conjugal.

\section{Une autonomie accrue}

Les femmes concernées ont pour caractéristique de ne pas avoir de partenaire de vie, en tout cas au moment de la migration. Elles sont soit encore célibataires à un âge considéré comme déjà avancé dans le pays d'origine (plus de trente ans), soit à la tête d'une famille dont elles assument seules la responsabilité, à commencer par celle de leurs enfants, mais pas uniquement. Dans bien des situations, elles contribuent à l'entretien de parents âgés ou d'autres parents proches (frères et sœurs). Demeurées seules suite à une séparation ou à un divorce, elles ont été contraintes de trouver des ressources leur permettant d'entretenir leurs enfants dans des pays où leur salaire ne suffisait pas. L'absence de conjoint ne signifie pas que le départ en migration s'effectue sans aucune négociation avec des membres de la famille, bien au contraire. Ils y consentiront d'autant plus facilement qu'une parente se trouve déjà à l'étranger.

Avec l'augmentation des revenus de la famille, l'acquisition de biens d'équipement et de consommation courante, l'envoi des enfants à l'université, la migration apparaît comme un facteur d'autonomisation pour les migrantes. Les conséquences de ce départ sont importantes au sein des foyers concernés, dans les pays d'origine, puisqu'elles conduisent à des inversions dans les rapports de dépendance et à une remise en cause de l'ascendant exercé par un père, un frère ou un oncle qui s'est substitué au conjoint défaillant. La dimension familiale est à prendre en compte lorsqu'il est question des mères de famille qui ont laissé des enfants au village ${ }^{23}$. De jeunes mères confient leurs propres enfants aux soins d'une parente proche, voire d'une autre femme qu'elles rémunèrent, pendant qu'elles mêmes sont recrutées pour s'occuper des enfants des autres ${ }^{24}$, devenant ainsi l'un des maillons de la global care chain. Ce type de circulation migratoire permet à ces migrantes d'assumer plus facilement leurs responsabilités maternelles, à la différence d'une migration d'installation. Dans le même temps, la migration assure un rôle de promotion sociale non seulement pour les migrantes, mais aussi et surtout pour leurs enfants. En ce sens, migrer n'est pas vital en soi, il s'agit d'une démarche fonctionnant comme une sorte d'assurance non pas contre la pauvreté en tant que telle, mais contre la précarité.

\footnotetext{
23 Quand ce sont de jeunes adultes, il s'agit de financer leurs études, voire leur mariage. Maurizio Ambrosini fait la même observation en ce qui concerne les femmes originaires d'Europe de l'Est en Italie (2005: 85).
}

24 Dans certains cas, la chaîne est encore plus poussée : par exemple, des recherches ont souligné I'existence de migrations de jeunes femmes indiennes peu qualifiées en Arabie saoudite où elles s'occupent des enfants de leurs compatriotes elles-mêmes salariées dans des hôpitaux (Catarino et Morokvasic, 2005). 
Dans ce contexte, les remises envoyées par les migrantes ${ }^{25}$ permettent de contrebalancer les reproches qui leur sont adressés en termes de " coût social " de la migration (absence, éloignement, etc.) dès lors que ces mères de famille deviennent le principal soutien financier de la cellule familiale,. Si " Analytically, monetary remittances represent long-distance social ties of solidarity, reciprocity, and obligation that bind migrants to their kin and friends accross statecontrolled national borders "(Guarnizo, 2003 : 671), dans le cas de migrations féminines, elles sont - encore plus fréquemment que pour les migrants masculins qui s'installent plus volontiers dans le pays d'immigration - mises au service de stratégies de mobilité sociale, avant tout pour les enfants (études, cours privés, etc.). L'étude de la " géométrie " des remises permet d'interroger le système de relations sociales plus large au sein duquel il est crucial de comprendre qui remet et qui reçoit, la situation familiale, personnelle et culturelle, ainsi que le contexte structurel (économique, social, politique) faisant également partie des variables décisionnelles (Lindley, 2007 : 13). C'est essentiellement par le biais financier que s'opèrent les reconfigurations de pouvoir au sein de la cellule familiale dans la mesure où les migrantes sont le plus souvent les seules pourvoyeuses. Cela se traduit par une participation accrue aux décisions prises au sein de la cellule familiale dans le pays d'origine, y compris lorsque les fonctions de chef de famille n'ont pas été transférées à un parent proche.

La littérature existante sur les mobilités féminines offre encore des conclusions contrastées, entre autonomisation/empowerment et stabilisation des rapports de genre (Morokvasic, 2010 : 105). Les données de terrain tendent, pour le sujet qui nous concerne, à abonder dans le sens d'une réelle autonomie des femmes concernées à l'égard de leur cercle d'origine (famille essentiellement). $\mathrm{Ce}$, justement parce qu'elles n'ont pu compter que sur elles-mêmes à tous les stades du processus. Tout d'abord, en finançant sur leurs économies le premier voyage, ou en contactant un prêt auprès d'une tierce personne. Par la suite, c'est grâce au capital d'informations réunies en amont et sur place qu'elles ont pu s'insérer dans les réseaux adéquats à leur arrivée. Une fois rodées à cette nouvelle vie, elles goûtent à une certaine forme d'indépendance à laquelle elles ne sont pas prêtes à renoncer, loin du qu'en-dira-t-on de la petite ville de province ou du village dont elles sont originaires. De ce point de vue, Istanbul, mégapole de quinze millions d'habitants, offre à la fois vie privée et anonymat. Nino, I'auxiliaire de vie géorgienne, comparait Ankara, où elle avait travaillé deux ans, et Istanbul : " À Ankara c'est moins bien, et puis il y a beaucoup de femmes voilées, moi j'apprécie beaucoup la vie à Kadiköy. ". Nino portait un regard très peu amène sur sa vie $d^{\prime}$ 'avant et sur son mariage. L'un dans l'autre, elle semblait considérer qu'il valait mieux être seule que mal accompagnée. Le départ de son mari lui avait permis de s'émanciper, fût-ce au prix de longues périodes de séparation avec ses enfants. Ne s'en laissant pas compter, elle avait même balayé d'un revers de la main les réticences de son frère, dont la propre situation professionnelle (il était sans emploi) et financière ne lui conférait, à ses yeux, qu'une autorité toute relative.

25 Rappelons qu'en 2009, les remises des migrants travaillant en Turquie s'élevaient à 141 millions de dollars, soit sept fois moins que les remittances sortantes, c'est-à-dire envoyées vers la Turquie par des émigrés turcs. Voir Banque mondiale : www.worldbank. org/prospects/migrationandremittances, page consultée le 3 décembre 2011. 
Le cas de Nelly que nous évoquions précédemment est quant à lui emblématique de la rupture occasionnée par la disparition d'une figure masculine tutélaire (en l'occurrence le père), événement à l'origine de la migration. Éduquée, divorcée et mère de deux grands adolescents, elle n'a pu se lancer dans la migration que suite au décès de son père en 2009, après une longue période d'autocensure. Quoi qu'elle affirme que l'" occasion ne s'était pas présentée avant ", il y a en fait tout lieu de penser que son père l'en aurait empêchée. Elle racontait que sa mère, institutrice de formation, $n^{\prime}$ avait jamais pu travailler car son mari " ne l'avait pas laissée ". Nelly se retrouvait seule avec ses deux enfants à charge car son ex conjoint " ne s'occupait pas de sa famille". Or la parente qui avait facilité sa venue et son installation à Istanbul s'y trouvait depuis plusieurs années, et lors de l'entretien Nelly n'a pas pu (voulu ?) éclaircir pourquoi elle n'avait pas saisi cette occasion plus tôt. En ce sens, le décès de son père a occasionné pour elle une véritable bifurcation biographique, c'est-àdire une " séquence d'action partiellement imprévisible [produisant] des effets durables " (Grossetti, 2006 : 11). Même s'il est probablement exagéré de soutenir qu'elle n'aurait jamais tenté l'aventure migratoire sans le décès de son père, on peut néanmoins supposer que cette disparition a rendu la situation matérielle de la famille plus délicate. En somme, aspiration individuelle et contexte familial auraient convergé.

Si la plupart des femmes rencontrées ont émigré pour des raisons économiques et familiales, certaines ont tout simplement saisi une opportunité inattendue. Par exemple Gayané, Arménienne âgée de 35 ans, a profité de ses congés pour rendre visite à des parents installés en Turquie depuis de nombreuses années. Lors de l'entretien (Istanbul, novembre 2013), elle travaillait comme plongeuse dans un restaurant d'Aksaray ${ }^{26}$. Elle déclarait ne pas avoir encore de plan précis pour l'avenir si ce n'est, à très court terme, profiter de son visa pour gagner l'argent qui lui permettrait d'améliorer son quotidien de fonctionnaire de la poste en Arménie. Certaines mobilités reposent en outre sur des réseaux familiaux qui plongent leurs racines loin dans I'histoire, à l'instar de cette quinquagénaire originaire de Tbilissi, mère d'un fils adulte et " vivant sans mari ". D’origine géorgienne par son père et arménienne par sa mère, elle a pu compter à son arrivée sur le soutien de parents originaires de $\mathrm{Kars}^{27}$. Contrairement aux autres migrantes évoquées précédemment, ces deux femmes libérées d'obligations familiales se sont quasiment lancées à l'aventure afin d'améliorer leur ordinaire. La migration représente pour elles un moyen de repartir sur de nouvelles bases.

Les migrations de type économique que nous avons évoquées jusqu'à présent reposent essentiellement sur des facteurs push, mais il n'en va pas forcément de même pour toutes les formes de mobilités des femmes ex-soviétiques en Turquie. Les mariages binationaux représentent une part non négligeable de ces

26 Quartier du district de Fatih, Aksaray se situe à l'extrémité du quartier de Sultanahmet. Il s'agit d'un important nœud de transports qui abrite en outre de nombreuses activités commerciales à destination d'une clientèle étrangère.

27 Ville de Turquie orientale qui a donné son nom à la région dont elle est la préfecture. La région de Kars se trouvait sous suzeraineté arménienne au Moyen Âge. Après avoir changé plusieurs fois de souveraineté entre 1918 et 1921, elle a finalement été intégrée à la Turquie par le traité de Kars en octobre 1921. 
flux, la présence d'un partenaire en Turquie étant alors évidemment un facteur pull lorsque la migration n'a pas préexisté à la rencontre.

\section{Roxelane, Natacha et les autres}

"Les Russes sont belles ". "Les Russes boivent beaucoup donc ils ne s'intéressent pas aux femmes ". "En Russie, les femmes dépassent les hommes "28. II s'agit là de commentaires exprimés, à un moment $\mathrm{T}$, à un chercheur sur son terrain qui reflètent, comme tout préjugé, I' "état cristallisé d'un imaginaire " (Guillaumin, $1972: 2$ ). Assénés sur un ton péremptoire, ils se caractérisent par leur résistance à la contradiction et émanent tant des individus que des institutions ou des médias. Le cas des "Natacha " est emblématique dans la mesure où il découle d'une situation socioéconomique - la présence en Turquie de prostituées originaires d'ex-URSS depuis le début des années 1990 - à l'origine de stéréotypes reposant sur un imaginaire ancré de longue date. II y a, bien sûr, l'image d'Épinal des esclaves enlevées en Russie, en Ukraine et au Caucase et devenues favorites du sultan, telle Roxelane (Hürrem, de son nom turc), l'épouse de Soliman le Magnifique, dont certains historiens pensent qu'elle aurait été d'origine non pas circassienne mais slave. Un feuilleton turc à grand succès (Muhtesem yüzyıl, Le siècle magnifique), tant en Turquie que dans les Balkans ${ }^{29}$ (Courrier international, 2012), relate cet épisode qui met en image l'espace complexe du harem où évoluaient nombre de femmes originaires de Russie, d'Ukraine et du Caucase.

Les unions binationales entre des ressortissants turcs et des ressortissantes russes ou ukrainiennes sont un terreau fertile pour les commentaires plus ou moins neutres découlant de préjugés et de stéréotypes bien ancrés de part et d'autre. Ces unions font partie des flux migratoires concernés sans être du reste incompatibles avec les thématiques évoquées précédemment, bien au contraire : si pour certaines femmes, l'union a préexisté à la venue en Turquie, pour d'autres elle s'inscrit dans un parcours qui a débuté par une migration économique. Les données de terrain indiquent une prééminence de la première configuration, favorisée par l'essor des moyens de communication, tel internet, qui rend les relations à distance plus aisées. Le profil des jeunes femmes originaires d'Ukraine et de Russie qui se marient actuellement en Turquie, ou qui y entretiennent une relation amoureuse, a également évolué. L'accusation plus ou moins fondée selon les cas de " mariage blanc " semble perdre de sa pertinence. Dans les années 1990, la conclusion d'unions de convenance était un moyen classique, pour les ressortissantes d'ex-URSS, de se maintenir sur le territoire turc et d'obtenir rapidement la citoyenneté turque. Des commerçantes à la valise ont ainsi été en mesure de créer leur propre entreprise plus facilement une fois naturalisées (Yükseker, 2003). Les autorités turques ont durci les conditions de naturalisation par le mariage, qui demeurent moins restrictives que dans d'autres pays, puisque les démarches peuvent être entamées après trois ans de vie commune seulement.

28 Nous avons entendu des commentaires identiques de la part d'immigrés sud-caucasiens au cours d'un terrain de thèse en Russie entre 2006 et 2009.

29 Voir "LaTurquie à la reconquête des Balkans ", Courrier international, n¹147, $25 / 10 / 2012$. 
Lorsqu'on évoque les mariages binationaux entre des ressortissants turcs et des ressortissantes d'ex-URSS, le caractère " mixte " de ces unions est un paramètre à prendre en compte. Il convient dès lors d'expliciter ce qu'on entend par l'expression " mariage mixte ". A minima, on peut considérer qu'il s'agit d'un " mariage entre personnes différentes ". Comment, alors, préciser ces différences, étant entendu que la réaction de l'environnement social est également une variable à prendre en compte ? Les couples concernés sont hétérogames dans la mesure où ils unissent deux conjoints qui diffèrent l'un de l'autre sur une au moins des grandes dimensions de l'existence : citoyenneté, lieu de vie, langue maternelle, religion, milieu social, âge. Dans notre cas, la dimension religieuse est pertinente puisque les femmes concernées sont originaires de Russie et d'Ukraine, plus rarement des pays ou régions de tradition musulmane du Caucase ou d'Asie centrale. Si l'on pose que "la relation d'amour est à la fois la plus simple, la plus profonde et la plus mystérieuse des relations humaines ", il faut également constater une forme de complexification lorsque le couple est mixte car, aux problèmes de compréhension et de communication avec l'autre qui se posent au sein de tous les couples, s'ajoutent plusieurs variables selon les situations : variable intercommunautaire, voire interétatique dans le cas qui nous intéresse, les deux individus ne relevant pas du même système juridique. Indépendamment des contraintes extérieures, les récits recueillis racontent tous plus ou moins la même réalité : les choix, les événements et les hasards de la vie qui conduisent un individu à sortir de son groupe social et culturel d'origine pour lier son destin à un autre groupe. À cet égard, les jeunes femmes rencontrées sont fréquemment déjà tournées vers l'international ou ont une appétence pour la différence ou pour l'étranger de longue date. Par exemple, cette jeune Russe originaire d'Ekaterinburg (ville de l'Oural), âgée de vingt-trois ans, qui avant de s'installer en Turquie a vécu en Italie, à Rome et dans les Pouilles, avec son petit ami italien. Ces différentes expériences ont dans bien des cas pour effet de conférer une relative maturité à ces jeunes femmes, qui se fixent en Turquie à la suite de leur mariage.

La fratrie du jeune homme, en fait une sœur, est fréquemment sollicitée pour servir d'intermédiaire. Ladite sœur, envoyée en éclaireur en quelque sorte, sera chargée de jauger sa potentielle belle-sœur, d'évaluer si elle est " comme il faut ", présentable à la famille. La cérémonie du mariage, qui pourrait a priori être source de désaccords et de disputes, est au contraire l'occasion pour le fiancé de préparer un événement qui paraîtra inoubliable pour sa future épouse. Les familles des deux parties sont rarement réunies, pour des questions pécuniaires essentiellement. Financer un voyage à l'étranger n'est guère envisageable pour ces familles russes et ukrainiennes, originaires de milieux modestes de province. Certains couples décident alors d'organiser une noce dans les deux pays, ce qui peut être l'occasion, pour les amis du marié, de se rendre en Ukraine. Irina raconte ainsi le déroulement de son mariage :

"Au début je ne voulais pas organiser de noce car c'était cher et trop d'organisation, mais mon mari a insisté en disant que sinon je le regretterai toute ma vie. Ça a été beaucoup de préparatifs et d'organisation, mais on a fait un mariage et en Ukraine et en Turquie. Avec les intimes, les très proches à chaque fois pour la cérémonie en elle-même, aucun de nos parents (sa belle-mère est veuve) n'était présent à chaque fois à cause du prix pour mes parents et de la santé de ma belle-mère, elle a de gros problèmes de dos. II n'y avait pas d'Ukrainiens en Turquie mais des amis de mon mari sont venus en Ukraine, 
où une limousine avait été louée pour cinq heures de balade et de photos dans la ville.

Et, on avait loué les services d'une interprète pour les invités turcs, elle leur a appris

la danse traditionnelle ukrainienne, ils ont beaucoup apprécié et ont été les derniers à partir, ils voulaient même rester et continuer la fête ! À Istanbul, mon mari avait loué une chambre à l'hôtel Hilton. C'était incroyable, très beau, avec une immense vitre coulissante dans la salle de bain. Bref, c'est un mariage tel que je l'avais rêvé." "

(Irina, Istanbul, janvier 2013)

Nombre de ces jeunes femmes ont pour point commun d'avoir une expérience amoureuse, voire une vie de couple, plus importante que leur partenaire en Turquie. Pour certaines, la rencontre fortuite avec leur futur conjoint fait suite à des démarches beaucoup plus organisées dans le but de contracter une union avec un étranger. Irina expliquait ainsi s'être un temps adjoint les services d'une agence matrimoniale par le biais de laquelle elle avait rencontré plusieurs hommes étrangers - dont un Américain - pour lesquels elle n'avait eu, comme elle le soulignait, " aucun coup de cœur ". Elle a finalement décidé de partir pour laTurquie afin de rejoindre son futur mari, rencontré dans une discothèque d'Odessa, " le jour même où l'autre (le citoyen américain) atterrissait en Ukraine afin de rencontrer d'autres fiancées potentielles ".

La question de la religion a été évoquée invariablement par presque toutes nos interlocutrices. Sans que ce sujet soit constitué en problème, il semble faire l'objet de négociations, ou plutôt de discussions, en amont du mariage. La crainte de devoir se convertir à l'islam apparaît relativement répandue chez les jeunes femmes rencontrées. Les relations avec la belle-mère sont également un sujet fréquemment abordé lors des entretiens ; nos interlocutrices faisant en l'occurrence le constat d'une absence de tension. La communication est cependant dans un premier temps rendue difficile par la non-maîtrise de la langue de l'autre - I'anglais étant la première langue privilégiée au moment de la rencontre- , ce qui ne va pas sans créer des malentendus. Irina se remémorait, en riant, son embarras le jour de son mariage, lorsque tous les membres de la famille de son mari étaient venus la féliciter personnellement et qu'elle s'était contentée de " sourire bêtement ". Elle parlait aussi de sa honte de ne pas comprendre ce que sa belle-mère lui disait lorsqu'elles étaient ensemble et se désolait du peu de progrès qu'elle effectuait en turc ${ }^{30}$. Elle relatait les pourparlers ayant présidé à son installation en Turquie, des sujets les plus banals (" J'ai quitté mes amis et ma famille pour lui, alors il peut bien supporter mes chats! ") aux plus sérieux (la religion, par exemple), donnant ainsi à voir le fragile équilibre qui s'instaure au quotidien, les interstices de négociation, soit l'ordinaire d'un couple lambda.

\section{Conclusion}

Étudier les migrations des femmes originaires de I'ex-URSS en Turquie permet tout à la fois d'appréhender dans leur diversité les mobilités occasionnées par un bouleversement géopolitique majeur, de battre en brèche les idées reçues quant aux notions de "Nord " et de "Sud ", et d'interroger certaines

30 La situation d'entretien a été favorable aux confidences car Irina se trouvait depuis trois mois seulement à Istanbul (en janvier 2013) et n'y connaissait personne. Les occasions de s'exprimer, sinon dans sa langue maternelle, du moins dans une langue - le russe - qu'elle maîtrisait tout autant se faisaient donc rares. 
représentations. Au niveau micro, la dichotomie traditionnelle entre " migrantes mères/filles/épouses de " et "migrantes indépendantes " demeure dans une certaine mesure opérante, dans une ville comme Istanbul où le secteur de la confection, qui s'appuie entre autres sur une myriade de petits ateliers, est pourvoyeur d'emplois à domicile pour certaines migrantes dont la mobilité s'inscrit dans une démarche familiale (Dedeoglu, 2011). Figures personnifiant habituellement le privé, le familial et l'émotionnel, les femmes sont parvenues, pour certaines, à retourner en leur faveur cet état de fait en mettant en exergue les qualités de care, de responsabilité et de compassion comme caractéristiques essentielles (Yuval-Davis, 1999 : 6). En situation d'immigration, ces qualités semblent propices à cantonner les migrantes à des secteurs d'activités considérés comme étant féminins. L'essentiel paraît alors se jouer dans les méandres des relations familiales, qui subissent une profonde reconfiguration en raison de l'autonomie acquise par celles qu'on appelle les mères transnationales.

\section{Références bibliographiques}

Abdelhafid Hammouche et Rude-Antoine Edwige Eds. (2006) Le couple : attention fragile, Hommes et migrations, 1262 (numéro spécial), 97 p.

Adelkhah Fariba et Bayart Jean-François (2007) Voyages du développement. Émigration, commerce, exil, Paris, Karthala, $368 \mathrm{p}$.

Ambrosini Maurizio (2005) L'immigration en Italie. Le cas de I'aide à domicile en Lombardie, Migrations société, 17 (102), pp. 71-88.

Andrijasevic Rutvica (2005) La traite des femmes d'Europe de l'Est en Italie. Analyse critique des représentations, Revue européenne des migrations internationales, 21 (1), [en ligne] consulté le 4 août 2012. URL : http://remi.revues. org/2354

Basak Serap Uzun (2011) Le retour des Turcs de l'étranger, 27 août, [en ligne] consulté le 30/01/2014. URL: http://ovipot.hypotheses.org/6474.

Basu Sipannita and Werbner Pnina (2009) Who Wants to Be a Millionnaire? Gendered Entrepreneurship and British South Asian Women in the Culture Industries, Revue européenne des migrations internationales, 25 (3), pp. 53-77.

Benhabib Seyla and Resnik Judith (2009) Migrations and Mobilities. Citizenship, Borders, and Gender, New York, New York University Press, 505 p.

Bilici Faruk (1994) Chocs et trocs en tout genre à la frontière turco-géorgienne, Panoramiques, 14 (2), pp. 167-171.

Bilici Faruk (2012) Sarp/Sarpi : la porte de l'intégration entre la Turquie et la Géorgie vingt ans après la chute de I'Union soviétique, Anatoli, 3, pp. 203-222.

Carling Jorgen, Menjivar Cecilia and Schmalzbauer Leah (2012) Central Themes in Transnational Parenthood, Journal of Ethnic and Migration Studies, 38 (2), pp. 191-217

Catarino Christine et Morokvasic Mirjana (2005) Femmes, genre, migration et mobilités, Revue européenne des migrations internationales, 21 (1), pp. 7-27.

Chimienti Milena et Földhàzi Ági (2008) Géographies du marché du sexe : entre dynamiques urbaines, économiques et politiques, Sociétés, 1 (99), pp. 79-90. 
Collet Beate et Philippe Claudine (2008) Mixités : variations autour d'une notion transversale, Paris, L'Harmattan, $288 \mathrm{p}$.

Courrier international (2012) La Turquie à la reconquête des Balkans, 1147, 25 octobre.

Cossée Claire, Miranda Adelina et Ouali Nouria (2012) Le genre au cœur des migrations, Paris, Éditions Petra, 336 p.

Dedeoglu Saniye (2011) Garment Ateliers and Women Workers in Istanbul: Wives, Daughters and Azerbaijani Immigrants, Middle Eastern Studies, 47 (4), pp. 663-674.

Donato M. Katharine et al. (2006) A Glass Half Full? Gender in Migration Studies, International Migration Review, 40 (1), pp. 3-26.

Erder Sema and Kaska Selmin (2003) Irregular Migration and Trafficking in Women:The Case of Turkey, International Organization for Migrations, 86 p.

Foucher Michel (2012) L'obsession des frontières, Paris, Perrin, 249 p.

Gender and Migration Revisited (2006) International Migration Review, 40 (1), $256 \mathrm{p}$.

Gil Françoise (2008) La prostituée, une invention sociale, Sociétés, 1 (99), pp. 21-32.

Grossetti Michel (2006) L'imprévisibilité dans les parcours sociaux, Cahiers internationaux de sociologie, 120, pp. 5-27.

Guerry Linda (2009) Femmes et genre dans I'histoire de l'immigration. Naissance et cheminement d'un sujet de recherche, Genre et histoire, [en ligne] consulté le 19/02/2014. URL : http://genrehistoire.revues.org/808.

Guillaumin Colette (1972) L'idéologie raciste, genèse et langage actuel, Paris-La Haye, Mouton, 247 p.

Guillemaut Françoise (2008) Femmes africaines. Migrations et travail du sexe, Sociétés, 1 (99), pp. 91-106.

Guyaux Anne et Delcroix Catherine (1992) Double mixte. La rencontre de deux cultures dans le mariage, Contradictions, 68 (numéro spécial), 132 p.

Halajkann Myriem (1994) Migration des femmes mauriciennes vers la France, Migrations Société, 6 (35), pp. 18-26

Içduygu Ahmet (2008) Rethinking Irregular Migration in Turkey: Some Demoeconomic Reflections, Consortium for Applied Research on International Migration, CARIM, 72, [en ligne] consulté le 20/02/2014. URL: http://cadmus.eui. eu/handle/1814/1011723p.

Kaya Ibrahim (2008) Circular Migration and Turkey : A Legal Perspective, CARIM, 37, [en ligne] consulté le 20/02/2014. URL: http://cadmus.eui.eu/bitstream/ handle/1814/8355/CARIM_AS\%26N_2008_37.pdf?sequence=13 p.

Kirisci Kemal (2008) Informal 'Circular migration' Into Turkey: The Bureaucratic and Political Context, CARIM, 21, [en ligne] consulté le 20/02/2014. URL: http://cadmus.eui.eu/bitstream/handle/1814/8342/CARIM_AS\%26N_2008_21. pdf?sequence $=1,16 \mathrm{p}$. 
Leandro Maria Engràcia (2006) Les flux migratoires des femmes portugaises et leur rôle dans les réseaux sociaux, Migrations Société, 18 (104), pp. 17-40.

Le Espiritu Yen (2005) Gender, Migration, and Work. Filipina Health Care Professionals to the United States, Revue européenne des migrations internationales, 21 (1), pp. 55-75, [en ligne] consulté le 23/08/2012. URL : http://remi. revues.org/2343.

Lim Lin Lean (2010) Traite, demande et marché du sexe, in Jules Falquet et al., Le sexe de la mondialisation, Paris, Presses de Sciences Po, pp. 165-178.

Lindley Anna (2007) The Early Morning Phonecall: Remittances from a Refugee Diaspora Perspective, Centre on Migration, Policy and Society, Working Paper, 47.

Mahler Sarah J. and Sabrina R. Pessar (2006), Gender Matters: Ethnographers Bring Gender from the Periphery toward the Core of Migration Studies, 40 (1), pp. 27-63.

Morokvasic Mirjana (2010) Le genre est au cœur des migrations, in Jules Falquet et al., Le sexe de la mondialisation, Paris, Presses de Sciences Po, pp. 105-120.

Neyrand Gérard, Philippe Claudine et Varro Gbarielle (1998), Liberté, égalité, Mixités conjugales. Une sociologie du couple mixte, Paris, Economica, 311 p.

Ottavio (D') Germana (2005) Va-et-vient : mobilité internationale des Polonaises dans I'Europe élargie, Migrations Société, 17 (2), pp. 89-103.

Péraldi Michel (2002) La fin des norias ? Réseaux migrants dans les économies marchandes en Méditerranée, Paris, Maisonneuve et Larose, 495 p.

Péraldi Michel (2009) Istanbul. Le commerce à la valise, face discrète de l'économie, Urbanisme, 369, pp. 67-68.

Percot Marie (2005) Les infirmières indiennes émigrées dans les pays du Golfe : de l'opportunité à la stratégie, Revue européenne des migrations internationales, 21 (1), [en ligne] consulté le 3/08/2012. URL : http://remi.revues.org/2340.

Piart Luisa (2012) Le lien entre le commerce à la valise et l'industrie de la confection à Istanbul, Anatoli, 3, pp. 23-40.

Potot Swanie (2005) La place des femmes dans les réseaux migrants roumains, Revue européenne des migrations internationales , 21 (1), [en ligne] consulté le 2/08/2012. URL: http://remi.revues.org/2335.

Sassen Saskia (2000) Cities in a World Economy, Londres, Thousand Oaks, 157 p.

Schmoll Camille (2005) Pratiques spatiales transnationales et stratégies de mobilité des commerçantes tunisiennes, Revue européenne des migrations internationales, 21 (1), pp. 131-154.

Tajfel Henri (1981) Human Groups and Social Categories. Studies in Social Psychology, New York, Cambridge University Press, 384 p.

Tarrius Alain (2002) La mondialisation par le bas. Les nouveaux nomades de l'économie souterraine, Paris, Éditions Balland, 220 p.

Tinguy (de) Anne (2004) La grande migration, Paris, Plon, 662 p.

Yuval-Davis Nira and Werbner Pnina (1999) Citizenship and Difference, New York, Zed books, $256 \mathrm{p}$. 
Yükseker H. Deniz (2003) Laleli-Moskova Mekigi. Kayitdisi Ticaret ve Cinsiyet Iliskileri [La navette Laleli-Moscou : le commerce à la valise et les relations de genre], Istanbul, Iletisim Yayinlari, 288 p.

Yükseker H. Deniz (2004) Trust and Gender in a Transnational Market: The Public Culture of Laleli, Istanbul, Public Culture, 16 (1), pp. 47-65. 


\section{Adeline Braux}

\section{: Au-delà des " Natacha " : les migrations féminines postsoviétiques à Istanbul}

Dans les années 1990 et au début des années 2000, la présence des "Natacha ", comme on appelle en Turquie les prostituées originaires de I'ex-URSS, avait défrayé la chronique et donné naissance à des stéréotypes tenaces sur les femmes originaires d'Europe de l'Est. L'entertainment business est cependant loin d'être le seul domaine d'activité économique des migrantes ex-soviétiques en Turquie. Parmi les secteurs économiques traditionnels investis par ces dernières, on trouve, dans le tertiaire, celui des services à la personne. Si le titre de l'article se propose d'aller au-delà des clichés véhiculés habituellement sur les femmes immigrées originaires de l'ex-URSS, il invite également à considérer, sinon sous un angle nouveau, du moins sous un angle plus large, l'épineuse question de la prostitution et de la traite des femmes. L'auteure s'intéresse aussi aux migrations dites "matrimoniales ", liées aux unions contractées entre ressortissants turcs et ressortissantes ukrainiennes et russes. Ce type d'union a pu par le passé déclencher des commentaires plus ou moins neutres, à présent, alors que la situation socioéconomique s'est améliorée en Ukraine et en Russie, on peut s'interroger sur la pertinence de la catégorie analytique du " mariage blanc".

\section{$\cdots$ Beyond "Natasha": Women Migrations from Post-Soviet Countries in Istanbul}

In the 1990s and early 2000s, the presence of "Natasha" - the name given in Turkey to prostitutes coming from Eastern Europe - made headlines and gave rise to persistent stereotypes about women from Eastern Europe in Turkey. Prostitution is however far from being the only economic activity of post-Soviet female migrants in this country. Among the traditional economic sectors female migrants invest in, the service sector, especially domestic help services has experienced sustainable growth. The title of the article proposes explicitly to go beyond the usual clichés associated with women from Eastern Europe and leads also to consider - if not from a new standpoint at least from a more open angle - the tricky issues of prostitution and trafficking in women. Most of the time indeed, this phenomenon is studied from the angle of the violence against women, and other aspects of the question are left aside. And, last but not least, we also intend to scrutinize the bi-national marriages contracted by Turkish citizens with women originating from Russia and Ukraine. These marriages are also part and parcel of migration flows between Turkey and former Soviet countries. Sometimes they trigger more or less neutral comments, disapproval, and criticism, which are based on deeply rooted prejudices and stereotypes on both sides. Now that the socio-economic situation in Russia and Ukraine has improved, the suspicion of a "paper marriage" seems not very consistent.

\section{Más allá de las «Natacha»: las migraciones femeninas postsoviéticas en Estambul}

Más allá de las "Natacha»: las migraciones femeninas postsoviéticas en Estambuldel ejército libanés en la entrada de un campo de refugiados, las rutas clandestinas hacia Grecia y las relaciones con las autoridades y algunos de los 
vecinos de la capital de este país. Durante aquellos tránsitos problemáticos, los refugiados-migrantes están obligados a ajustarse a la situación en el marco de interacciones asimétricas: intercambios con un soldado, un "pollero", un guardia fronterizo o inclusive con ciudadanos de Atenas. Entonces el autor intenta describir las respuestas que los refugiados aportan a las asignaciones identitarias y a los llamamientos a justificar su presencia en estos cruces. Además, busca sacar a la luz el punto de vista que ellos desarrollan más ampliamente acerca de su postura, entre refugios y migraciones. 